\title{
AN EXAMPLE FOR FACTORIZATION THEORY IN BANACH ALGEBRAS
}

\author{
P. G. DIXON
}

\begin{abstract}
It has been conjectured that, if $A$ is a separable Banach algebra in which every element factorizes (i.e. for every $x \in A$ there exist $y, z \in A$ with $x=y z$ ), then every pair of elements in $A$ has a common factor. An example is given of a commutative, separable Banach algebra $A$ where the set $M$ of elements of $A$ which factorize is of codimension one in $A$ and there exists a pair of elements of $M$ with no common factor in $A$.
\end{abstract}

It has been conjectured [2, 5.3(iii)] that, if $A$ is a separable Banach algebra in which every element factorizes, then every pair of elements of $A$ has a common factor. This conjecture remains open. The purpose of this note is to show that if the conjectured result is true, it will depend crucially on all the elements factorizing. We give an example of a commutative, separable Banach algebra $A$ in which the set $M$ of elements which factorize is of codimension one in $A$, but such that there exists a pair of elements of $M$ with no common factor (indeed many such pairs will exist). It is hoped that this example will also be found interesting for its use (unusual in this context) of the notion of topological degree.

To clarify definitions: we shall say that an element $x \in A$ factorizes if there exist $y, z \in A$ with $x=y z$. We say that $x_{1}, x_{2} \in A$ have a common factor $y \in A$ if there exist $z_{1}, z_{2} \in A$ with $x_{1}=y z_{1}, x_{2}=y z_{2}$. We write

$$
A^{2}=\left\{x_{1} y_{1}+\cdots+x_{n} y_{n}: x_{i}, y_{i} \in A(1 \leq i \leq n), n=1,2,3, \ldots\right\} \text {. }
$$

We denote by $\mathbf{C}$ the complex plane and by $\mathbf{C}^{*}$ the Riemann sphere (the one-point compactification of $\mathbf{C})$. We write $\Delta=\{z \in \mathbf{C}:|z|<1\}, \bar{\Delta}=\{z \in \mathbf{C}:|z| \leq 1\}$ and $\partial \Delta=\{z \in \mathbf{C}:|z|=1\}$.

Our example is the uniform algebra $A$ consisting of all continuous functions $f: \mathbf{C}^{*} \rightarrow \mathbf{C}$ such that $f$ is analytic on $\Delta$ and $f(0)=0$. Let $M=\left\{f \in A: f^{\prime}(0)=\right.$ $0\}$.

THEOREM. The algebra $A$ is a separable uniform algebra. The subspace $A^{2}=$ $M$ is of codimension one and every element of $M$ factorizes (in $A)$. However, there exists a pair of elements of $M$ with no common factor (in $A$ ).

Proof. The algebra $A$ is separable because $\mathbf{C}^{*}$ is metrizable. The key fact about $A$ is that every $f \in A$ has at least one zero in $\mathbf{C}^{*} \backslash \Delta$. To see this, suppose $f \in A$ with no zeros in $\partial \Delta$. Then, since $f$ is analytic on $\Delta$ with $f(0)=0$, the degree of $f$ on $\partial \Delta$ (i.e. the topological degree of the mapping $f /|f|: \partial \Delta \rightarrow \partial \Delta$ ) is strictly positive. But, since $f$ is continuous on $\mathbf{C}^{*} \backslash \Delta$, this forces $f$ to have a zero in $\mathbf{C}^{*} \backslash \bar{\Delta}$.

Received by the editors September 4, 1981.

1980 Mathematics Subject Classification. Primary $46 \mathrm{H} 05$.

Key words and phrases. Banach algebra, factorization. 
Now let $h_{1}, h_{2} \in M$ with no common zero in $\mathbf{C}^{*} \backslash \Delta$. (Such elements are easily constructed.) Then $h_{1}, h_{2}$ can have no common factor $f \in A$, since any zero of $f$ in $\mathbf{C}^{*} \backslash \Delta$ would be a common zero of $h_{1}$ and $h_{2}$.

It is clear that $M$ is of codimension one and that $A^{2} \subseteq M$. The hardest part of the proof is to show that every element $h \in M$ can be written as $h=f g$ with $f, g \in A$. The proof of this divides into two cases.

The easier case is when $h$ has no zero on $\partial \Delta$. The degree argument given above implies that $h\left(z_{0}\right)=0$ for some $z_{0} \in \mathbf{C}^{*} \backslash \bar{\Delta}$. By using a homeomorphism of $\mathbf{C}^{*} \backslash \Delta$ which fixes $\partial \Delta$ and sends $z_{0}$ to $\infty$, we may assume, without loss of generality, that $z_{0}=\infty$. We then define

$$
f(z)= \begin{cases}z & (z \in \bar{\Delta}), \\ \left(|z|^{-1}+\left(1-|z|^{-1}\right)|h(z)|^{1 / 2}\right) z /|z| & (z \in \mathbf{C} \backslash \Delta), \\ 0 & (z=\infty),\end{cases}
$$

and $g(z)=h(z) / f(z)(z \in \mathbf{C}), g(\infty)=0$. One easily verifies that $f, g \in A$.

The second case is when $h\left(z_{1}\right)=0$ for some $z_{1} \in \partial \Delta$. Let $B$ be the uniform algebra consisting of all continuous, complex-valued functions on $C^{*}$ which are analytic on $\Delta$ and vanish at $z_{1}$ (but not necessarily at 0 ). Then $B$ has a bounded approximate identity $\left(e_{n}\right)$ given by

$$
\begin{array}{ll}
e_{n}(z)=1-2^{-n}\left(z z_{1}^{-1}+1\right)^{n} & (z \in \bar{\Delta}), \\
e_{n}(z)=e_{n}\left(\bar{z}^{-1}\right) & \left(z \in \mathbf{C}^{*} \backslash \bar{\Delta}\right) .
\end{array}
$$

We may therefore apply Cohen's Factorization Theorem [1, $\S 11$, Corollary 11] to $B$ and deduce that $h=f g$ for some $f, g \in B$. If $f(0)=g(0)=0$, then $f, g \in A$ and the result is proved. Otherwise, suppose $g(0) \neq 0$. Then $f(0)=f^{\prime}(0)=0$, since $h \in M$. Let $\varsigma:\left(\mathbf{C}^{*} \backslash \Delta\right) \backslash\left\{z_{1}\right\} \rightarrow \partial \Delta \backslash\left\{z_{1}\right\}$ be a retraction (i.e. $\zeta$ is continuous and restricts to the identity on $\left.\partial \Delta \backslash\left\{z_{1}\right\}\right)$. We then have $h=f_{1} g_{1}$, where

$$
\begin{aligned}
& f_{1}(z)= \begin{cases}0 & (z=0), \\
f(z) / z & (z \in \bar{\Delta} \backslash\{0\}), \\
f(z) / \varsigma(z) & \left(z \in \mathbf{C}^{*} \backslash \bar{\Delta}\right),\end{cases} \\
& g_{1}(z)= \begin{cases}z g(z) & (z \in \bar{\Delta}), \\
\varsigma(z) g(z) & \left(z \in \mathbf{C}^{*} \backslash \bar{\Delta}\right) .\end{cases}
\end{aligned}
$$

It is easily verified that $f_{1}, g_{1} \in A$.

\section{REFERENCES}

1. F. F. Bonsall and J. Duncan, Complete normed algebras, Ergebnisse der Math. und ihrer Grenzgebiete, bd. 80, Springer-Verlag, Berlin, 1973.

2. P. G. Dixon, Automatic continuity of positive functionals on topological involution algebras, Bull. Austral. Math. Soc. 23 (1981), 265-281.

Department of Pure Mathematics, The University, Sheffield, S3 7RH, ENGLAND 